\title{
Dynamic Asset Allocation and Downside-Risk Aversion
}

\author{
Arjan Berkelaar Roy Kouwenberg
}

Econometric Institute Report EI 2000-12/A

March 27, 2000

\author{
Econometric Institute \\ Department of Finance \\ Faculty of Economics \\ Faculty of Economics \\ Erasmus University Rotterdam \\ Erasmus University Rotterdam \\ P.O.Box 1738, 3000 DR Rotterdam \\ P.O.Box 1738, 3000 DR Rotterdam \\ The Netherlands \\ The Netherlands
}

\begin{abstract}
This paper considers dynamic asset allocation in a mean versus downside-risk framework. We derive closed-form solutions for the optimal portfolio weights when returns are lognormally distributed. Moreover, we study the impact of skewed and fat-tailed return distributions. We find that the optimal fraction invested in stocks is V-shaped: at low and high levels of wealth the investor increases the stock weight. The optimal strategy also exhibits reverse time-effects: the investor allocates more to stocks as the horizon approaches. Furthermore, the investment strategy becomes more risky for negatively skewed and fat-tailed return distributions.
\end{abstract}

Keywords: optimal asset allocation, downside-risk.

JEL Classifications Codes: G12 


\section{Introduction}

A growing number of practitioners are using downside-risk measures in various portfolio management applications. Due to the concern of regulating authorities and the need for establishing risk management systems at banks and institutional investors, the popularity of such downsiderisk measures has grown considerably. Partly, this can be attributed to the appealing notion that investors care mainly about the risk associated with downside movements and that upside potential should not be penalized. ${ }^{1}$ The most famous example is value-at-risk based risk management which has become an industry standard by regulation (Jorion 1997). Besides value-at-risk, other popular downside-risk measures include expected shortfall and downside deviation.

Despite its popularity and widespread use little is known about the implications of downsiderisk management for asset allocation. One of the early references on the merits of downside risk measures for asset allocation is Markowitz (1959). Markowitz (1959) realized the drawbacks of variance as a measure of risk, as it penalizes both upside and downside movements in the portfolio value equally. He proposed semivariance as a more appropriate risk measure, measuring risk as deviations below the mean only. However, Markowitz was not able to resolve the difficulties of a mean-semivariance framework due to the non-differentiability involved.

Recent literature on the use of downside risk measures for portfolio theory still relies on simulations and computational results (Harlow 1991, Sortino \& van der Meer 1991, Markowitz, Todd, Xu \& Yamane 1993) and focus on one-period buy-and-hold strategies. In the traditional financial-economic literature, on the other hand, closed-form solutions have been derived for dynamic asset allocation models. The main focus, however, is on portfolio selection under power utility (Samuelson 1969, Merton 1969) and the impact of the predictability of asset returns (Brennan, Schwartz \& Lagnado 1997, Kim \& Omberg 1996, Campbell \& Viceira 1999, Sørensen 1999). The issue of downside risk management is largely ignored in this literature.

In this paper we study dynamic asset allocation in a mean versus downside-risk framework. The investor makes a trade-off between expected return and downside-risk. We derive closed-form solutions for optimal asset allocation in this setting. Additionally, we consider the impact of negatively skewed and fat-tailed return distributions. In particular, we address the following questions:

1. What is the optimal investment strategy in a mean downside-risk framework?

2. Does the optimal portfolio choice of a downside-risk averse investor exhibit time diversification effects?

3. How does a downside-risk averse investor behave when confronted with negatively skewed and fat-tailed asset returns?

Our results are as follows. First, under general security price processes, we show that investors

\footnotetext{
${ }^{1}$ We like to remark that downside risk aversion is closely related to loss aversion and prospect theory advocated by Kahneman \& Tversky (1979), as they both assume that people treat gains and losses differently.
} 
who care mainly about falling below the target (e.g. probability of a loss) and investors who care more about the magnitude of deviations below the target (e.g. downside variance) behave quite differently. The first group of investors desires full insurance at intermediate wealth levels against falling below the threshold and exhibit gambling behavior at low levels of wealth. The second group of investors do not desire full insurance: they accept small deviations below the threshold and behave more prudent.

An interesting result is that we can decompose the optimal strategies in derivatives contracts. This provides a clear interpretation of the investment strategy without assuming specific asset return distributions. The first group of investors desires to hold two binary options on the growth optimal portfolio. A binary option can be interpreted as a probability maximizing strategy. The second group holds a binary option and a butterfly spread option on the growth optimal portfolio. We also point to a drawback of the mean downside-risk framework, caused by risk neutrality above the target. This permits the investor to accept excessive gambles.

Second, when asset prices follow a geometric Brownian motion, we derive closed-form solutions for the optimal portfolio weights. The optimal fraction invested in stocks as a function of wealth displays a V-shaped pattern. When confronted with gains, risk neutrality induces the investor to increase his exposure to stocks. When confronted with losses the investor also increases his stock weight. For small lower partial moments (e.g. probability of a loss) this effect is caused by risk seeking behavior below the target. For larger lower partial moments (e.g. downside variance) this can attributed to the increasing relative risk aversion property of the corresponding utility function.

An important issue is how investors should allocate to risky assets depending on their horizon. Our closed-form solutions enable us to study these time-effects. It is a well-known result that investors with power utility allocate a constant fraction of wealth to risky assets regardless of their investment horizon. When investors are downside-risk averse this result does no longer hold. In general, downside-risk aversion causes reversed time-effects, i.e. when confronted with a shorter horizon the investor allocates more heavily to stocks. The rationale behind the reversed time effect is risk neutrality when confronted with gains and risk seeking behavior $(0 \leq \gamma<1)$ or increasing relative risk aversion $(\gamma=2)$ when confronted with losses.

Finally, we are interested in the effect of skewness and kurtosis in asset returns on the optimal portfolio choice under downside-risk aversion. In the standard case of constant relative risk aversion or for mean-variance optimizers, skewed and fat-tailed return distributions do not affect the optimal allocation to stocks. When employing downside-risk measures this is no longer true. The impact of skewness and kurtosis is quite large and can not be ignored. Again we find striking results: expected loss and probability of loss minimizers $(0 \leq \gamma \leq 1)$ increase the stock weight as the return distribution becomes more negatively skewed. Moreover, investors in a mean versus downside risk framework invest more in stocks when confronted with fat tails, due to risk neutrality above the threshold.

In conclusion, despite the popularity of downside-risk as a measure for investment risk and a guideline for optimal investment strategies, a mean versus downside risk framework implies many peculiarities: 
1. Both at low and high levels of wealth the investor gambles by investing more in risky assets;

2. Downside-risk aversion gives rise to reversed time effects: confronted with a shorter horizon the investor invests more in risky assets;

3. Confronted with negatively skewed and fat-tailed returns the investor invests more in risky assets.

It is questionable whether investors and practitioners are aware of the gambling effects prevalent in the use of downside-risk measures. It should be noted that optimal strategies derived under downside-risk aversion do indeed minimize the downside-risk of an investment portfolio. However, this is accompanied with gambling strategies that may not be approved of by regulators or management. In practice short selling constraints will certainly restrain investors from (extremely) risky investment strategies. However, regardless of short selling constraints, the nature of the optimal investment strategies remains the same.

Surprisingly, there is little previous research on optimal portfolio choice under downside-risk aversion. Basak \& Shapiro (1999) study investment behavior in the traditional setting of power utility subject to a value-at-risk constraint. Although they only focus on expected utility with value-at-risk as a side-constraint, their findings confirm the gambling effects established in this paper. Dert \& Oldenkamp (1997) consider a one-period discrete state model aiming at maximizing expected return subject to a guaranteed return constraint. They also find a gambling effect and refer to it as a casino effect. However, they do not provide closed-form solutions for the optimal portfolio choice.

This paper is organized as follows. In Section 2 we introduce our continuous-time economy and discuss the mean versus downside-risk framework. In Section 3 we specify the agent's optimization model and consider the optimal strategies under general price processes. We also derive closed-form solutions in this section for the optimal fraction invested in risky assets when asset prices follow geometric Brownian motions and interest-rates and the market price of risk are constant. Section 4 studied the optimal portfolio choice problem under downside-risk aversion when asset returns are skewed and fat-tailed. Section 5 concludes this paper.

\section{Economic Setting}

\subsection{The Economy}

In this section we formulate our economy and the dynamic investment problem. We consider a finite-horizon, $[0, T]$, economy. ${ }^{2}$ We assume that the investor trades $K+1$ assets continuously in a market without transaction costs. The zero-th asset is a riskless money market account $S_{0}(t)$ :

$$
d S_{0}(t)=r(t) S_{0}(t) d t
$$

\footnotetext{
${ }^{2}$ All stated processes are assumed to be well-defined and satisfy the appropriate regularity conditions. For technical details the reader is refered to Karatzas \& Shreve (1998).
} 
The prices of the remaining assets $S=\left\{S_{k}(t)\right\}_{k=1}^{K}$ follow Ito processes with drift rate $\mu_{k}(t)$ and volatility $\sigma_{k}(t)$ :

$$
d S_{k}(t)=\mu_{k}(t) S_{k}(t) d t+\sigma_{k}(t) S_{k}(t) d B_{t}, \quad k=1, \ldots, K,
$$

where the interest rate $r(\cdot)$, the drift rates $\mu(\cdot)$ and the volatility matrix $\sigma(\cdot)$ are adapted process (possibly path-dependent).

In order to meet his investment goals the investor chooses a portfolio consisting of the $K$ risky assets and the riskless money market account. We denote the fraction invested in risky asset $k$ at time $t$ by $w_{k}(t)$ and the fraction invested in the riskless asset at $t$ by $w_{0}(t)$. For any selffinancing portfolio the wealth $W_{t}$ of the investor can be expressed as the following stochastic process (using vector notation):

$$
d W_{t}=r(t) W_{t} d t+(\mu(t)-\iota r(t))^{\prime} w(t) W_{t} d t+W_{t}(w(t) \sigma(t))^{\prime} d B_{t} .
$$

where we substituted $w_{0}(t)=1-\sum_{i} w_{i}(t)$. The initial wealth of the investor is denoted by $W_{0}$.

Markets are assumed to be complete, implying the existence of a unique state price density (or pricing kernel) $\xi_{t}$, given by

$$
\xi_{t}=\exp \left(-\int_{0}^{t} r(s) d s\right) Z_{t}
$$

where $Z_{t}$ denotes the Radon-Nikodym derivative of a change of probability measure defined by

$$
Z_{t}=\frac{d Q}{d P}=\exp \left(-\frac{1}{2} \int_{0}^{t}\|\kappa(s)\|^{2} d s-\int_{0}^{t} \kappa(s)^{\prime} d B_{s}\right)
$$

and $\kappa(t)=\sigma^{-1}(t)(\mu(t)-\iota r(t))$ denotes the market price of risk process. We assume that there are no redundant assets, and consequently $\sigma$ is an invertible matrix, as markets are complete.

Alternatively, we may write the state price density process as

$$
\frac{d \xi_{t}}{\xi_{t}}=-r(t) d t-\kappa(t)^{\prime} d B_{t}, \xi_{0}=1
$$

The state price density process (or pricing kernel) will play an important role in deriving the optimal trading strategies. Merton (1990) has shown that the inverse of the pricing kernel is equal to the value of the growth-optimum portfolio. The growth optimum portfolio, $\pi_{t}$, is the strategy that maximizes the expected growth rate of the portfolio (or the average continuously compounded return on the portfolio), i.e. $\pi_{t}=\arg \max _{\pi_{t}} E_{t}\left(\log \left(W_{T}\right)\right)$. Moreover, this portfolio is mean-variance efficient. When the investment opportunity set is constant and utility is concave and differentiable, any optimal portfolio can be represented in terms of simple combinations of the growth optimum portfolio and the riskless asset (so-called two fund separation).

\subsection{Downside-Risk Aversion}

Many portfolio management applications are based on the traditional mean-variance framework (Markowitz 1952). Mean-variance theory can either be justified by assuming quadratic utility 
or by assuming normal returns. Quadratic utility exhibits increasing relative and absolute risk aversion, properties that are considered unrealistic by many financial economists, as they imply that investors invest more in risky assets as their wealth decreases. Moreover, there is a vast amount of empirical evidence that stocks returns may not be normally distributed. Finally, employing variance as a risk-measure both upside and downside movements in the portfolio value are penalized equally.

Markowitz (1959) already realized the drawbacks of variance as a measure of risk, and proposed semivariance as a more appropriate risk measure, measuring risk as deviations below the mean only. However, he was not able to resolve the difficulties of a mean-semivariance framework due to the non-differentiability involved. In a recent paper Markowitz et al. (1993) return to this issue and rely on computational techniques to derive the optimal asset allocation in a meansemivariance framework. In this paper we are able to derive closed-form solutions for a more general mean versus downside-risk framework.

Downside-risk measures penalize only negative returns relative to a given benchmark $\theta$. Moreover, downside-risk measures allow for asymmetric return distributions and are consistent with more general utility functions (Fishburn 1977). A widely applied class of downside-risk measures is based on the so-called lower partial moments (LPMs) as introduced by Bawa \& Lindenberg (1977). Investors make a trade-off between mean and downside-risk, where risk is associated with the partial moments of the return distribution of the investment portfolio, measured with regard to the target $\theta$, i.e.

$$
R_{\gamma}(\theta)=E\left([\max \{\theta-x, 0\}]^{\gamma}\right)=\int_{-\infty}^{\theta}(\theta-x)^{\gamma} d F(x), \quad \gamma \geq 0
$$

where $F(x)$ denotes the probability distribution function over portfolio returns $x$.

The reference point $\theta$ distinguishes gains from losses and serves as a benchmark for investing. Commonly used definitions are the expected portfolio return, the riskfree rate, or a certain target rate of return. The benchmark could be time-varying, e.g. in index tracking, or represent the liabilities of an institutional investor, e.g. in asset liability management studies. In this paper we assume that $\theta$ is constant (and $\theta \geq 1$ ), and interpret it as a benchmark wealth level. We could also incorporate a stochastic benchmark, e.g. following a geometric Brownian motion. However, the results and conclusions do not change qualitatively.

The class of LPMs (4) includes measures such as shortfall probability $(\gamma=0)$, expected shortfall $(\gamma=1)$, and downside-variance $(\gamma=2)$. Variance is only a special case of (4) when returns are normally distributed. Letting $\gamma \rightarrow \infty$ it can be shown that the risk associated with worst possible outcomes is a limiting case (Fishburn 1977). Finally, we like to mention that Value-atRisk is closely related to the class of risk measures (4). VaR is the maximum amount of money that you may lose with a given probability, over a certain time period. In our situation, the $\alpha \%$-VaR corresponds to the benchmark level $\hat{\theta}(\alpha)$ such that $R_{0}(\hat{\theta}(\alpha))=1-\alpha$.

In a mean-variance framework investors make a trade-off between expected return and risk (as measured by variance), i.e. they maximize $\mu(x)-\lambda \sigma^{2}(x)$, where $\mu$ denotes the mean and $\sigma$ the variance of the return on a portfolio $x$. In the mean versus downside risk framework 
investors make a similar trade-off, however, only negative returns relative to the benchmark $\theta$ are considered as risk. They maximize:

$$
E(x)-A E\left([\max \{\theta-x, 0\}]^{\gamma}\right),
$$

where $a$ and $A$ are constants, and $A$ denotes the risk aversion of the investor. In this paper we refer to investors who maximize (5) as downside-risk averse investors.

It is not difficult to derive that downside-risk averse investors who maximize (5) can be modeled as expected utility maximizers with the following utility function:

$$
U(x)= \begin{cases}x-A(\theta-x)^{\gamma} & x \leq \theta \\ x & x>\theta\end{cases}
$$

where $A \geq 0$ to ensure that $U(\cdot)$ is an increasing function, and $\gamma>0$. Often downside-risk is measured in terms of portfolio returns, here we assume that the investor only measures downsiderisk at the investment horizon, and therefore we formulate the utility function (6) in terms of terminal wealth.

As mentioned above, the class of utility functions (6) has a clear interpretation in terms of related risk measures. Defining risk as negative preferences gives rise to the following risk measures: probability of a loss $(\gamma=0)$, expected losses $(\gamma=1)$, semivariance or downside variance $(\gamma=2)$, and worst possible outcome $(\gamma=\infty)$. Note that when $\gamma$ is small, the investor cares about small deviations below the target, while for large $\gamma$ the investor is more concerned with larger deviations below the target, as he penalizes these deviations more heavily.

When $\gamma<1$ the investor is locally risk-seeking over losses. In this case the investor is willing to gamble in an attempt to minimize the extent to which his return falls short of $\theta$. If $\gamma>1$ the investor is locally risk-averse over losses. The relative risk-aversion function below the threshold $\theta$ is given by:

$$
R(x)=-\frac{x U^{\prime \prime}(x)}{U^{\prime}(x)}=\frac{A x \gamma(\gamma-1)(\theta-x)^{\gamma-2}}{1+A \gamma(\theta-x)^{\gamma-1}}
$$

It is not difficult to conclude that when $1<\gamma \leq 2$ the investor displays increasing relative risk aversion over losses. When $\gamma>2$, this no longer holds, and the relative risk aversion function is both increasing and decreasing on its domain.

Utility function (6) is linear above $\theta$, implying risk neutrality. The optimization problem therefore becomes unbounded, and a proper analysis is not possible. We can resolve this by introducing a satiation level $\bar{W}$ : utility remains constant for wealth in excess of $\bar{W}$. Consequently the investor invests fully in the riskfree asset as wealth at time $t$ exceeds $\bar{W} e^{-r(T-t)}$. Note that the original case can be retrieved by letting $\bar{W} \rightarrow \infty$. In the next section we study the optimal portfolio choice problem when investors care about the downside-risk of their asset value. We consider investment behavior under downside-risk aversion under general price processes, and interpret the results in terms of derivative contracts. 
Dynamic Asset Allocation and Downside-Risk Aversion

\section{Portfolio Optimization Under Downside-Risk Aversion}

\subsection{Agent's Optimization Model}

In this section we solve the portfolio optimization problem of a downside risk averse investor and discuss and analyze the properties of the solution. We make no assumptions on the distribution of the asset returns throughout this section as we derive the optimal wealth profile of a downsiderisk averse investor at his investment horizon. This general derivation provides insight in the structure of the optimal strategy regardless of distributional assumptions. In the next section we specialize to the case where asset prices follow geometric Brownian motions with constant interest rate and market price of risk and we study some of the analytic properties of the optimal strategies in more detail.

We explicitly incorporate a non-negativity constraint on the investor's wealth, as the utility function does not exhibit infinite marginal utility at zero wealth. The investor aims at solving the following dynamic portfolio problem:

$$
\begin{array}{ll}
\max & E\left[U\left(W_{T}\right)\right] \\
\text { s.t. } & d W_{t}=r W_{t} d t+(\mu-\iota r)^{\prime} w_{t} W_{t} d t+\sigma^{\prime} w_{t} W_{t} d B_{t} \\
& W_{t} \geq 0, \forall t \in[0, T]
\end{array}
$$

The martingale methodology allows the problem to be restated as the following static optimization problem.

$$
\begin{array}{ll}
\max & E\left[U\left(W_{T}\right)\right] \\
\text { s.t. } & E\left[\xi_{T} W_{T}\right] \leq \xi_{0} W_{0} \\
& W_{T} \geq 0
\end{array}
$$

The assumption of complete markets, a constant opportunity set and the absence of exogenous sources of income (e.g. labor income) ensures that the optimally invested wealth will never reach zero before time $T$ (Cox \& Huang 1989). As a result it suffices to restrict terminal wealth only.

Proposition 1 characterizes the optimal terminal wealth for a downside-risk averse investor when $0 \leq \gamma \leq 1$ (including expected loss and probability of loss).

Proposition 1 The time $T$ optimal wealth of a downside-risk averse investor with $0 \leq \gamma \leq 1$ is:

$$
W(T)= \begin{cases}\bar{W} & \text { if } \xi_{T}<\xi \\ \theta & \text { if } \bar{\xi}<\bar{\xi}_{T}<\bar{\xi} \\ 0 & \text { if } \bar{\xi}_{T}>\bar{\xi}\end{cases}
$$

where $\underline{\xi}=\frac{1}{y}$ and $\bar{\xi}=\frac{1}{y}+\frac{A}{y} \theta^{\gamma-1}$

Figure 1(a) displays the optimal terminal wealth for a downside risk averse investor with $0 \leq \gamma \leq$ 1 as a function of $\xi_{T}$. In good states $\left(\right.$ low $\xi_{T}$ ) the investor attains satiation, and consequently 
wealth is at the upper bound $\bar{W}$. In intermediate states $\left(\xi_{T}\right.$ between $\underline{\xi}$ and $\left.\bar{\xi}\right)$ the investor keeps his wealth at the benchmark level $\theta$. In bad states (high $\xi_{T}$ ) the investor ends up with zero wealth. When first order risk aversion becomes more pronounced, or equivalently downside-risk aversion increases ( $A$ increases) the intermediate states region expands at the cost of the bad states region. As $\gamma$ increases, the investor becomes less risk seeking and consequently $\bar{\xi}$ increases.

The optimal terminal wealth of a downside-risk averse investor with $0 \leq \gamma \leq 1$ can be interpreted as an investment in two binary options on the growth optimal portfolio with value $Z_{t}:=1 / \xi_{t}$. The first binary option pays $\theta$, with a strike price equal to $1 / \bar{\xi}$. This binary option is the optimal strategy for an investor that maximizes the probability that terminal wealth exceeds $\theta$ (or equivalently the investor minimizes the probability of falling short of $\theta$ ). The second binary option pays $\bar{W}-\theta$, with a strike price equal to $1 / \underline{\xi}$.

For downside-risk measures with $\gamma>1$ (e.g. downside variance) the optimal terminal wealth profile is quite different as the investor, in this case, becomes risk averse over losses, and additionally cares more about larger deviations below $\theta$. Proposition 2 characterizes the optimal terminal wealth for a downside-risk averse investor when $\gamma>1$.

Proposition 2 The time $T$ optimal wealth of a downside-risk averse investor with $\gamma>1$ is:

$$
W(T)= \begin{cases}\bar{W} & \text { if } \xi_{T}<\underline{\xi} \\ \theta-\left(\frac{y \xi_{T}-1}{A \gamma}\right)^{1 /(\gamma-1)} & \text { if } \underline{\xi}<\xi_{T} \leq \bar{\xi} \\ 0 & \text { if } \xi_{T}>\bar{\xi}\end{cases}
$$

where $\underline{\xi}=\frac{1}{y}$ and $\bar{\xi}=\frac{1}{y}+\frac{A \gamma}{y} \theta^{\gamma-1}$

Figure 1(b) displays the optimal terminal wealth for a downside risk averse investor with $\gamma>1$ as a function of $\xi_{T}$. In good states (low $\xi_{T}$ ) the investor attains satiation, and consequently keeps wealth at the upper bound $W_{T}$. In intermediate states $\left(\xi_{T}\right.$ between $\underline{\xi}$ and $\left.\bar{\xi}\right)$ terminal wealth is always below the threshold $\theta$. In bad states (high $\xi_{T}$ ) the investor ends up with zero wealth. The parameters of the utility function have a clear impact on the interval of intermediate states $[\xi, \bar{\xi}]$. If downside-risk aversion increases (i.e. $A$ increases) the intermediate states region expands. If the investor desires a larger target return $\theta$ this region also expands.

The investor has to make a trade-off between the amount of insurance at intermediate states and the size of the intermediate states region. A downside-risk averse investor with $0 \leq \gamma \leq 1$ fully insures against intermediate states while the investor with $\gamma>1$ only partially insures against these intermediate states. It should be noted that, when $\gamma>1$, terminal wealth is a convex function of the pricing kernel at intermediate states. Therefore, as $\gamma$ increases the investor desires less insurance, while at the same time the intermediate region expands. This can be understood by realizing that as $\gamma$ increases the investor is more concerned with large deviations below $\theta$ and consequently strives to avoid ending up with zero wealth at the horizon. For small $\gamma$ the investor is mostly concerned with the failure to meet the target. In the extreme case when $\gamma \rightarrow \infty$ the investor never ends up with zero wealth as $\bar{\xi} \rightarrow \infty$, and faces a constant payoff for $\xi_{T}>\underline{\xi}$ equal to $\theta-1$ (with $\theta \geq 1$ ). 
Again, the optimal strategy can be decomposed into an investment in two derivative contracts. The first option is a bull spread on the growth optimal portfolio $Z_{t}:=1 / \xi_{t}$ with exercise prices $1 / \bar{\xi}$ and $1 / \xi$. The bull spread consists of a long position in a call option stroke at $1 / \bar{\xi}$ and a short position in a call option stroke at $1 / \xi .^{3}$ An investor entering into a bull spread beliefs that markets will rise. The bull spread limits both the investor's upside potential and his downsiderisk. It should be noted that the payoff of the bull spread between $1 / \bar{\xi}$ and $1 / \underline{\xi}$ is nonlinear. The second option is a binary option on the growth optimal portfolio paying $\bar{W}-\theta$ with strike price equal to $1 / \underline{\xi}$, to gain additional upside.

Proposition 1 and Proposition 2 also demonstrate a drawback of the mean versus downside-risk framework. As the utility function is risk neutral above the target, the investment behavior is dominated by risk neutrality for large wealth levels. This implies gambling behavior. Consider the following situation. Assume that the initial wealth satisfies $W_{0}>\theta e^{-r T}$. By investing a part of his wealth $\left(\theta e^{-r T}\right)$ in the riskfree asset, the investor can always ensure that terminal wealth exceeds $\theta$. The remaining wealth is used to gamble as the investors behavior exhibits risk neutrality. Similar effects were found by Dert \& Oldenkamp (1997) in a one-period discrete time model. They termed this phenomenon the casino effect.

In our continuous-time model this effect is also present. Note that when wealth exceeds $\theta$ the investor behaves as a probability maximizer to attain the arbitrary upper bound $\bar{W}$. We only introduced the arbitrary upper bound $\bar{W}$ to arrive at a sensible policy and avoid unbounded solutions, due to risk-neutrality. As $\bar{W}$ is arbitrary, the investor bets on a favorable realization of $\xi_{T}$ yielding a high expected wealth level that occurs with a very small probability only. Letting $\bar{W} \rightarrow \infty$ this effect becomes clear: the probability of reaching infinite wealth is zero, while expected wealth equals infinity.

\subsection{Properties of the Investment Strategy}

In this section we derive closed-form solutions for the optimal portfolio strategy under downsiderisk aversion, when asset prices follow geometric Brownian motions. We assume that interest rates and the market price of risk are constant. The optimal portfolios can either be characterized in feedback form or in timeseries form (Dybvig 1995). The feedback form stresses the dependence of the optimal portfolio on the state variables and is common when applying dynamic programming techniques (Merton 1969). The timeseries form stresses the dependence of the optimal portfolio on the random uncertainty (i.e. the pricing kernel $\xi_{t}$ ), and is common when applying the martingale methodology (Cox \& Huang 1989).

As we assume that interest-rates and the market price of risk are constant and that the prices of the risky assets follow geometric Brownian motions, the pricing kernel $\xi_{T}$ is lognormally distributed. We focus on two distinct cases in this section: downside-risk aversion with $0 \leq \gamma \leq 1$ (including shortfall probability and expected shortfall) and downside-risk aversion with $\gamma=2$ (downside variance). We derive closed-form solutions and consider time effects on the demand for risky assets. In the next section we study the impact of skewed and fat-tailed return distributions.

\footnotetext{
${ }^{3}$ Alternatively, one may use put options to create the bull spread synthetically.
} 


\section{Case I: Shortfall Probability and Expected Shortfall}

We first consider the case of downside-risk aversion when $0 \leq \gamma \leq 1$. As we discussed in Section 2 special cases are shortfall probability $(\gamma=0)$ and expected shortfall $(\gamma=1)$. Proposition 3 characterizes the optimal portfolio when $0 \leq \gamma \leq 1$. The optimal portfolio is given in timeseries form.

Proposition 3 Assume that the investor is downside-risk averse with $0 \leq \gamma \leq 1$. Then

(i) The time $t$ optimal wealth is given by

$$
W(t)=(\bar{W}-\theta) e^{-r(T-t)} N\left(d_{1}(\underline{\xi})\right)+\theta e^{-r(T-t)} N\left(d_{1}(\bar{\xi})\right)
$$

where $N(\cdot)$ denotes the cumulative normal distribution function, $\underline{\xi}=\frac{1}{y}, \bar{\xi}=\frac{1}{y}+\frac{A}{y} \theta^{\gamma-1}$,

$$
d_{1}(x)=\frac{\log \left(\frac{x}{\xi_{t}}\right)+\left(r-\frac{1}{2}\|\kappa\|^{2}\right)(T-t)}{\|\kappa\| \sqrt{(T-t)}}
$$

(ii) The fraction of wealth invested in the risky assets is

$$
w(t)=\frac{\left(\sigma^{\prime}\right)^{-1} \kappa}{W(t)}\left(\frac{(\bar{W}-\theta) e^{-r(T-t)} \phi\left(d_{1}(\underline{\xi})\right)+\theta e^{-r(T-t)} \phi\left(d_{1}(\bar{\xi})\right)}{\|\kappa\| \sqrt{(T-t)}}\right)
$$

Note that $\gamma$ only influences $\bar{\xi}$. Furthermore, it should be noted that the fraction of wealth invested in the risky assets tends to zero as wealth goes to zero (to avoid bankruptcy) and as wealth goes to infinity (as the investor attains satiation).

Figure 2 displays the optimal intermediate wealth and the fraction invested in stocks for a downside-risk averse investor with $\gamma=0$ (e.g. probability of loss). We assume that there is one risky asset (stocks) only and that the investment horizon is one year. From Figure 2(a) we see that the investor's wealth is a smoothed version of the terminal wealth profile of Figure 1(a). In intermediate states the investor starts to insure himself. Figure 2(b) shows that the fraction invested in stocks is V-shaped for intermediate states. In good states (low $\xi_{t}$ ) the investor reduces his exposure to stocks as he attains satiation at $\bar{W} \exp (-r(T-t))$.

\section{Case II: Downside-Variance}

We now study the case of an investor making a trade-off between mean and downside variance. This investor cares more about larger deviations below the target and is risk averse over losses. Proposition 4 characterizes the optimal portfolio when $\gamma=2$. The optimal portfolio is given in timeseries form.

Proposition 4 Assume that the investor is downside-risk averse with $\gamma=2$. Then 
(i) The time $t$ optimal wealth is given by:

$$
\begin{aligned}
W(t)= & (\bar{W}-\bar{\theta}) e^{-r(T-t)} N\left(d_{1}(\underline{\xi})\right)+\bar{\theta} e^{-r(T-t)} N\left(d_{1}(\bar{\xi})\right) \\
& -\frac{y \xi_{t}}{2 A} e^{\Gamma(t)}\left(N \left(d_{2}(\bar{\xi})-N\left(d_{2}(\underline{\xi})\right)\right.\right.
\end{aligned}
$$

where $N(\cdot)$ denotes the cumulative normal distribution function, $\underline{\xi}=\frac{1}{y}, \bar{\xi}=\frac{1}{y}+\frac{A \gamma}{y} \theta^{\gamma-1}, \bar{\theta}=$ $\theta+\frac{1}{2 A}$

$$
\begin{aligned}
d_{1}(x) & =\frac{\log \left(\frac{x}{\xi_{t}}\right)+\left(r-\frac{1}{2}\|\kappa\|^{2}\right)(T-t)}{\|\kappa\| \sqrt{(T-t)}} \\
d_{2}(x) & =d_{1}(x)-\|\kappa\| \sqrt{(T-t)} \\
\Gamma(t) & =-2 r(T-t)+\|\kappa\|^{2}(T-t)
\end{aligned}
$$

(ii) The fraction of wealth invested in the risky assets is

$$
\begin{aligned}
w(t)= & \frac{\left(\sigma^{\prime}\right)^{-1} \kappa}{W(t)}\left(\frac{(\bar{W}-\bar{\theta}) e^{-r(T-t)} \phi\left(d_{1}(\underline{\xi})\right)}{\|\kappa\| \sqrt{(T-t)}}+\frac{\bar{\theta} e^{-r(T-t)} \phi\left(d_{2}(\bar{\xi})\right)}{\|\kappa\| \sqrt{(T-t)}}\right. \\
& \left.-\frac{y \xi_{t} e^{\Gamma(t)}}{2 A}\left(\frac{\phi\left(d_{2}(\bar{\xi})\right)-\phi\left(d_{2}(\underline{\xi})\right)}{\|\kappa\| \sqrt{(T-t)}}-\left(N\left(d_{2}(\bar{\xi})\right)-N\left(d_{2}(\underline{\xi})\right)\right)\right)\right)
\end{aligned}
$$

Note that the fraction of wealth invested in the risky assets tends to zero as wealth goes to zero (to avoid bankruptcy) and as wealth goes to infinity (as the investor attains satiation).

Figure 3 displays the optimal intermediate wealth and the fraction invested in stocks for a downside-risk averse investor with $\gamma=2$. The optimal fraction invested in stocks exhibits a $\mathrm{V}$-shaped pattern again for intermediate states. In good states (low $\xi_{t}$ ) the investor reduces his exposure to stocks as he attains satiation at $\bar{W} \exp (-r(T-t))$. Focusing on intermediate states, we may discern the differences between the strategy for $0 \leq \gamma \leq 1$ and $\gamma>1$ as discussed in the previous section.

As the downside-risk averse investor with $0 \leq \gamma \leq 1$ demands full protection at intermediate states at the investment horizon, he dedicates more aggressively to stocks at intermediate states. The downside-risk averse investor with $\gamma>1$, on the other hand, more gradually increases his exposure to stocks at intermediate states. In good states the behavior of both investors is similar. This becomes more clear if we consider the optimal fraction invested in stocks in feedback form. Figure 4 displays the optimal fraction invested in stocks as a function of wealth for both investors.

At high wealth levels the behavior of both investors is similar as they are both risk neutral above the benchmark level $\theta$ and reach satiation at $\bar{W} \exp (-r(T-t))$. Risk neutrality prescribes a full commitment to stocks as they provide a higher return. As the investors attain satiation they reduce their exposure to stocks for higher wealth levels. For low wealth levels both investors invest more heavily in stocks, however the expected shortfall and probability of loss minimizers behave more aggressively. When $0 \leq \gamma<1$ the investor becomes risk-seeking below $\theta$, explaining the aggressive investment behavior for low wealth levels. As utility function (6) displays 
increasing relative risk aversion below $\theta$ for $1<\gamma \leq 2$ the fraction invested in stocks increases as wealth decreases.

We may also calculate the indirect utility function even though it does have the desired derivatives to satisfy the Hamilton-Jacobi-Bellman equation of dynamic programming. The indirect utility function for $\gamma=2$ is given by

$$
\begin{aligned}
J\left(\xi_{t}, t\right)= & (\bar{W}-\bar{\theta}) N\left(d_{1}(\underline{\xi})+\sigma\right)+\bar{\theta} N\left(d_{1}(\bar{\xi})+\sigma\right)-A \theta^{2} N\left(-d_{1}(\bar{\xi})-\sigma\right) \\
& -\frac{y^{2} \xi_{t}^{2}}{4 A} e^{\Gamma(t)}\left(N\left(d_{2}(\bar{\xi})\right)-N\left(d_{2}(\underline{\xi})\right) .\right.
\end{aligned}
$$

Figure 5 shows a graph of the indirect utility function as a function of wealth. The indirect utility function is increasing relative risk averse for low levels of wealth (explaining why the optimal fraction invested in stocks increases as wealth decreases) and decreasing relative risk averse for high levels of wealth. For extremely high wealth levels the relative risk aversion function is increasing again as the investor attains satiation.

An interesting issue is the impact of the investment horizon on the demand for risky assets. When investor preferences exhibit constant relative risk aversion (CRRA) it is a well known result that the fraction invested in stocks is independent of the time horizon (Samuelson 1969, Merton 1969). When relative risk aversion is non constant this no longer holds. Assuming asset returns are lognormally distributed, decreasing relative risk aversion implies time diversification, i.e. an investor invests more in stocks when confronted with a longer investment horizon. Increasing relative risk aversion implies a reverse time diversification effect. For a recent discussion we refer to Kritzman (1998).

When the investor is downside-risk averse the investment horizon also has an impact on the demand for risky assets. Figure 6 displays the optimal fraction invested in stocks for different horizons. At large and small levels of wealth the optimal policy of the investor exhibits a reverse time-diversification effect. The reverse time-diversification effect at low wealth levels can be attributed to the increasing relative risk aversion property of the utility function at low wealth levels. The reverse time diversification effect at high wealth levels can be attributed to risk neutrality above the target. Only at a small region of intermediate wealth levels the optimal policy of the investor exhibits time diversification.

\subsection{Momentum and Portfolio Insurance Strategies}

In the previous sections we characterized the optimal strategies under downside-risk aversion. In this section we consider the number of shares purchased or sold as the underlying asset price changes. This provides some additional insight into the optimal trading strategies when investors are downside-risk averse. We assume again that interest-rates and the market price of risk are constant, and that the prices of the risky assets follow geometric Brownian motions. Assuming that there is only one risky asset (e.g. stocks), with price $S_{t}$ at time $t$, the pricing kernel $\xi_{t}$ can be expressed as a function of the stock price, as follows:

$$
\xi_{t}=B(t) S_{t}^{-(\mu-r) / \sigma^{2}}
$$


where $B(t)=\exp \left\{\frac{(\mu-r)\left(\mu+r-\sigma^{2}\right)}{2 \sigma^{2}} t\right\}$. This allows us to rewrite the optimal terminal wealth profile derived in the previous section as a function of the stock price.

We first consider the strategy of a HARA investor with utility

$$
U\left(W_{T}\right)=\frac{1-\alpha}{\alpha}\left(\frac{A W_{T}}{1-a}+\eta\right)^{\alpha} .
$$

The optimal terminal wealth for this investor is given by:

$$
W(T)=-\frac{\eta(1-a)}{A}+\frac{1-a}{A}\left(\frac{y C(T) S_{T}}{A}\right)^{\kappa / \sigma(1-\alpha)} .
$$

where $\kappa$ denotes the Sharpe ratio. Consequently, when $\kappa / \sigma(1-\alpha)>1$ the optimal strategy is convex (portfolio insurance strategy), and when $\kappa / \sigma(1-\alpha)<1$ the optimal strategy is concave (momentum strategy). When $\alpha>1$ the utility function exhibits increasing relative risk aversion and the optimal strategy is always a momentum (or reversal) strategy. When $1-\kappa / \sigma<\alpha<1$, the optimal strategy is a portfolio insurance strategy, and when $\alpha<1-\kappa / \sigma$ the optimal strategy is a momentum strategy.

Let us consider the strategy of a downside-risk averse investor now. For a downside-risk averse investor with $\gamma>1$ we obtain

$$
W(T)= \begin{cases}\bar{W} & \text { if } S_{T}>\bar{S} \\ \theta-\left(\frac{y B(T) S_{T}^{-(\mu-r) / \sigma^{2}}-1}{A \gamma}\right)^{1 /(\gamma-1)} & \text { if } \bar{S}<S_{T} \leq \underline{S} \\ 0 & \text { if } S_{T}<\underline{S}\end{cases}
$$

where $\underline{S}=\left(\frac{B(T)}{\bar{\xi}}\right)^{\sigma^{2} /(\mu-r)}$ and $\bar{S}=\left(\frac{B(T)}{\underline{\xi}}\right)^{\sigma^{2} /(\mu-r)}$. It is not difficult to show that for a downside-risk averse investor with $1<\gamma \leq 2$ terminal wealth is a concave function of the stock price between $\underline{S}$ and $\bar{S}$. When $\gamma>2$ this no longer holds and the optimal terminal wealth may be both convex and concave between $\underline{S}$ and $\bar{S}$. Consequently, when $1<\gamma \leq 2$ the investor follows a reversal strategy: selling high, buying low.

Figure 7 displays the number of stocks purchased as a function of the stock price. The initial stock price at $t=0$ equals $\$ 1$. The number of stocks purchased as the stock price increases is M-shaped. At low stock prices the investor buys stocks (or reversely sells stocks as prices drop to avoid bankruptcy), at intermediate stock price levels the investor is willing to sell stocks as he follows a reversal strategy (due to concavity), if the stock price goes up even further the investor starts buying again as he becomes risk neutral until he attains satiation and is willing to sell again. This illustrates the peculiarities and subtleties involved in using downside-risk aversion.

\section{Downside-Risk Aversion and Higher Order Moments}

In the previous section we considered the optimal investment policy of a downside-risk averse investor when asset returns are lognormally distributed, or equivalently when the continuously 
compounded returns are normally distributed. A common argument for the use of downsiderisk measures is that asset returns are negatively skewed and have fat tails. An analysis of the optimal investment strategy for downside-risk averse investors would therefore be incomplete without addressing this issue. In this section we study the impact of skewness and kurtosis on the demand for risky assets when investor are downside-risk averse.

When asset prices follow geometric Brownian motions, the continuously compounded returns are normally distributed. In order to incorporate skewness and kurtosis, one would have to specify alternative stochastic processes for the asset prices, such as jump processes (e.g. the variance-gamma processes of Madan \& Seneta (1990) or the hyperbolic model Eberlein, Keller \& Prause (1998)). The problem with these discontinuous processes is that markets become incomplete. This complicates the martingale methodology employed in this paper. ${ }^{4}$ We rather rely on a polynomial expansion of the normal density function, known as the Gram-Charlier expansion.

The appealing feature of the Gram Charlier expansion is that we do not need to make distributional assumptions which may be hard to justify. The Gram Charlier expansion is a simple expansion of the $(\log )$ normal density function, allowing us to incorporate skewness and kurtosis. This approach should be considered as an approximation technique, yielding additional insight, as we are able to derive closed-form solutions. The Gram Charlier expansion has also been used in the option pricing literature under similar assumptions (Longstaff 1995).

The Gram-Charlier expansion was first introduced in finance by Jarrow \& Rudd (1982). It provides a parsimonious representation of a distribution with skewness and kurtosis, and generates an approximate density function for a standardized random variable. For the lognormal density function, the Gram-Charlier expansion is given by

$$
f(z ; \beta, \delta)=\left(1+\beta\left(z^{3}-3 z\right)+\delta\left(z^{4}-6 z^{2}+3\right)\right) \phi(z),
$$

where $z=(\log (x)-\mu) / \sigma, \beta=\zeta / 6, \delta=\chi / 24$ and $\zeta, \chi$ denote skewness and kurtosis of $\log (x)$ respectively, and $\phi(\cdot)$ denotes the standard normal density function. Equation (15) is often viewed as an approximation to an arbitrary density function with nonzero higher moments, however, for moderate values of $\zeta$ and $\chi$ it is a density function in itself.

In our analysis we consider a Gram-Charlier expansion of the density function of the pricing kernel. Therefore, $\zeta$ and $\chi$ denote the skewness and kurtosis of the pricing kernel respectively. Assuming the existence of one risky asset only, $\zeta$ and $\chi$ also denote the skewness and kurtosis of the return on this risky asset. ${ }^{5}$ There is a vast amount of empirical evidence that asset returns are negatively skewed and fat-tailed. Fama (1965) was the first to show that stock returns (at a daily basis) exhibit fat tails. Sample estimates of skewness for stock index returns tend to be negative, while sample estimates of excess kurtosis for stock returns on indexes are positive.

Given the empirical evidence, it is important to study the effect of skewed and fat-tailed asset returns on the demand for risky assets. Obviously, this demand is affected by the preferences of

\footnotetext{
${ }^{4}$ The HJB approach can certainly not be used for these discontinuous processes.

${ }^{5}$ It is not difficult to show that if $X, Y$ are random variables such that $Y=a X+b$, then skew $(Y)=\operatorname{skew}(X)$ and $\operatorname{kurt}(Y)=\operatorname{kurt}(X)$.
} 
the investor, given by his utility function. When preferences are represented by a power utility function or for mean-variance optimizers and when interest rates and the market price of risk are constant, it can be demonstrated that skewness and kurtosis do not influence the optimal portfolio choice. This investor invests a constant fraction of wealth in risky assets, depending on the mean and covariance of asset returns only.

When the investor is downside-risk averse this is no longer true. The demand for risky assets under downside-risk aversion depends on the skewness and kurtosis of the asset returns. Proposition 5 characterizes the optimal portfolio choice for a downside-risk averse investor with $0 \leq \gamma \leq 1$ when asset returns exhibit skewness and kurtosis.

Proposition 5 Assume that the investor is downside-risk averse with $0 \leq \gamma \leq 1$. Let $\beta$ and $\delta$ denote scaled skewness and kurtosis respectively. Then

(i) The time $t$ optimal wealth is given by:

$$
W(t)=(\bar{W}-\theta) e^{-r(T-t)} G_{1}(\underline{\xi}, \sigma)+\theta e^{-r(T-t)} G_{1}(\bar{\xi}, \sigma)
$$

where $\underline{\xi}=\frac{1}{y}, \bar{\xi}=\frac{1}{y}+\frac{A}{y} \theta^{\gamma-1}$

$$
\begin{aligned}
d_{1}(x) & =\frac{\log \left(\frac{x}{\xi_{t}}\right)+\left(r-\frac{1}{2}\|\kappa\|^{2}\right)(T-t)}{\|\kappa\| \sqrt{(T-t)}} \\
\alpha(x) & =d_{1}(x)+\|\kappa\| \sqrt{(T-t)} \\
G_{i}(x, s) & =N\left(d_{i}(x)\right) Q_{1}(s, \beta, \delta)-\phi\left(d_{i}(x)\right) Q_{2}(\alpha(x), s, \beta, \delta) \\
Q_{1}(s, \beta, \delta) & =1+\beta s^{3}+\delta s^{4} \\
Q_{2}(\alpha, s, \beta, \delta) & =\beta\left[(\alpha+s)^{2}-\alpha s-1\right]+\delta\left[\alpha\left((\alpha+s)^{2}-\alpha s-3\right)+s\left(s^{2}-1\right)\right]
\end{aligned}
$$

$N(\cdot)$ denotes the cumulative standard normal distribution function and $\phi(\cdot)$ denotes the standard normal density function, and $s=\|\kappa\| \sqrt{(T-t)}$.

(ii) The fraction of wealth invested in the risky assets is

$$
\left.\left.w_{t}=\frac{\left(\sigma^{\prime}\right)^{-1} \kappa}{W(t)}\left(\frac{e^{-r(T-t)}(\bar{W}-\theta)}{\|\kappa\| \sqrt{(T-t)}} \Psi(\alpha(\underline{\xi}), s, \beta, \delta)\right)+\frac{\theta e^{-r(T-t)}}{\|\kappa\| \sqrt{(T-t)}} \Psi(\alpha(\bar{\xi}), s, \beta, \delta)\right)\right)
$$

where $\phi(\cdot)$ denotes the standard normal density function and

$$
\begin{aligned}
\Psi(\alpha, s, \beta, \delta) & =\phi(\alpha-s)\left(Q_{1}(s, \beta, \delta)-Q_{2}^{\prime}(\alpha, s, \beta, \delta)+(\alpha-s) Q_{2}(\alpha, s, \beta, \delta)\right) \\
& =\phi(\alpha-s)\left(1+\beta\left(\alpha^{3}-3 \alpha\right)+\delta\left(\alpha^{4}-3 \alpha^{2}-3 \alpha+3\right)+3 \delta s(1-\alpha)\right)
\end{aligned}
$$

Note that the solutions when asset returns are lognormally distributed are a special case of the solutions presented in Proposition 5 when $\beta=0$ and $\delta=0$. Figure 8 shows the effect of skewness and kurtosis on the optimal fraction invested in stocks for $\gamma=0$.

Figure $8(\mathrm{a})$ shows the effect of negative skewness. At low levels of wealth the investor invests more in stocks, as he is risk seeking, when confronted with negative skewness. At intermediate 
levels of wealth the investor invests less in stocks, as he desires more insurance (staying above the threshold). In good states the investor allocates more to stocks again as risk neutrality dominates his behavior. Figure 8(b) shows the effect of excess kurtosis. At most levels of wealth the investor invests more in stocks, when confronted with fat tails. This is caused by risk seeking behavior below $\theta$ and risk neutrality above $\theta$.

Proposition 6 presents closed-form solutions for a downside risk averse investor with $\gamma=2$ when asset returns exhibit skewness and excess kurtosis.

Proposition 6 Assume that the investor is downside-risk averse with $\gamma=2$. Let $\beta$ and $\delta$ denote scaled skewness and kurtosis respectively. Then

(i) The time t optimal wealth is given by:

$$
W(t)=(\bar{W}-\bar{\theta}) e^{-r(T-t)} G_{1}(\underline{\xi}, s)+\bar{\theta} e^{-r(T-t)} G_{1}(\bar{\xi}, s)-\frac{y \xi_{t}}{2 A} e^{\Gamma(t)}\left(G_{2}(\bar{\xi}, 2 s)-G_{2}(\underline{\xi}, 2 s)\right)
$$

where $\underline{\xi}=\frac{1}{y}, \bar{\xi}=\frac{1}{y}+\frac{A \gamma}{y} \theta^{\gamma-1}, \bar{\theta}=\theta+\frac{1}{2 A}$

$$
\begin{aligned}
d_{1}(x) & =\frac{\log \left(\frac{x}{\xi_{t}}\right)+\left(r-\frac{1}{2}\|\kappa\|^{2}\right)(T-t)}{\|\kappa\| \sqrt{(T-t)}} \\
d_{2}(x) & =d_{1}(x)-\|\kappa\| \sqrt{(T-t)} \\
\alpha(x) & =d_{1}(x)+\|\kappa\| \sqrt{(T-t)} \\
\Gamma(t) & =-2 r(T-t)+\|\kappa\|^{2}(T-t) \\
G_{i}(x, s) & =N\left(d_{i}(x)\right) Q_{1}(s, \beta, \delta)-\phi\left(d_{i}(x)\right) Q_{2}(\alpha(x), s, \beta, \delta) \\
Q_{1}(s, \beta, \delta) & =1+\beta s^{3}+\delta s^{4} \\
Q_{2}(\alpha, s, \beta, \delta) & =\beta\left[(\alpha+s)^{2}-\alpha s-1\right]+\delta\left[\alpha\left((\alpha+s)^{2}-\alpha s-3\right)+s\left(s^{2}-1\right)\right]
\end{aligned}
$$

$N(\cdot)$ denotes the cumulative standard normal distribution function and $\phi(\cdot)$ denotes the standard normal density function, and $s=\|\kappa\| \sqrt{(T-t)}$.

(ii) The fraction of wealth invested in the risky assets is

$$
\begin{aligned}
w_{t}=\frac{\left(\sigma^{\prime}\right)^{-1} \kappa}{W(t)} & \left.\left(\frac{e^{-r(T-t)}(\bar{W}-\bar{\theta})}{\|\kappa\| \sqrt{(T-t)}} \Psi(\alpha(\underline{\xi}), s, \beta, \delta)\right)+\frac{\bar{\theta} e^{-r(T-t)}}{\|\kappa\| \sqrt{(T-t)}} \Psi(\alpha(\bar{\xi}), s, \beta, \delta)\right) \\
& \left.-\frac{y \xi_{t}}{2 A} e^{\Gamma(t)}\left(\frac{\Psi(\alpha(\bar{\xi}), 2 s, \beta, \delta)-\Psi(\alpha(\underline{\xi}), 2 s, \beta, \delta)}{\|\kappa\| \sqrt{(T-t)}}-\left(G_{2}(\bar{\xi}, 2 s)-G_{2}(\underline{\xi}, 2 s)\right)\right)\right)
\end{aligned}
$$

where $\phi(\cdot)$ denotes the standard normal density function and

$$
\begin{aligned}
\Psi(\alpha, s, \beta, \delta) & =\phi(\alpha-s)\left(Q_{1}(s, \beta, \delta)-Q_{2}^{\prime}(\alpha, s, \beta, \delta)+(\alpha-s) Q_{2}(\alpha, s, \beta, \delta)\right) \\
& =\phi(\alpha-s)\left(1+\beta\left(\alpha^{3}-3 \alpha\right)+\delta\left(\alpha^{4}-3 \alpha^{2}-3 \alpha+3\right)+3 \delta s(1-\alpha)\right)
\end{aligned}
$$

Figure 9 displays the optimal fraction invested in stocks (assuming that only one risky asset is available) when the returns on stocks are negatively skewed and have fat tails. Comparing 
Figure 8 with Figure 9 it is clear that at low levels of wealth skewness and kurtosis have less impact on the optimal portfolio choice for an investor concerned with downside variance than for an investor focusing on shortfall probability. This can be understood by realizing that an investor with $\gamma<1$ is risk seeking below $\theta$. At higher levels of wealth the effects are comparable as both strategies are driven by risk neutrality.

Figure 9 shows the effect of negative skewness. At intermediate and high levels of wealth the impact of skewness and kurtosis is most pronounced. At high levels of wealth the investor invests more in stocks when confronted with negative skewness, due to risk neutrality. At intermediate levels of wealth the investor invests less in stocks when confronted with negative skewness, this can be understood by realizing that downside-risk aversion with $\gamma=2$ is consistent with an investor exhibiting (positive) skewness preference. In other words this investor dislikes negative skewness and therefore, invests less in stocks. At low levels of wealth the impact of negative skewness can be ignored. Both at intermediate and high levels of wealth the investor invests more in stocks as he faces fat tails. At low levels of wealth the impact of kurtosis can be ignored.

\section{Conclusions}

In this paper we studied investment behavior in a mean versus downside-risk framework. We derived closed-form solutions for the optimal portfolio choice when returns are lognormally distributed and when returns exhibit skewness and kurtosis. The optimal fraction invested in stocks is V-shaped. Both for low and high levels of wealth the investor desires a large exposure to stocks. Investors caring mainly about falling below the target $(0 \leq \gamma \leq 1)$ behave differently from investors caring more about the magnitude of losses below the target $(\gamma>1)$. The first group of investors desires full insurance at intermediate levels of wealth as they are averse against falling below the target. The second group of investors do not desire full insurance but care more about large deviations below the target.

Under downside-risk aversion time-effects have a large effect on the demand for risky assets. Both at large and small levels of wealth downside-risk aversion implies reverse time-effects: the investor invests more in risky assets as his horizon is shorter. Only at a small range of intermediate wealth levels the optimal strategy exhibits time diversification: the investor invests less in risky assets as his horizon is shorter. We also studied the number of shares purchased or sold as a function of the asset price. The optimal number of shares is M-shaped. At low stock prices the investor buys stocks, at intermediate stock price levels the investor is willing to sell stocks as he follows a reversal strategy, if the stock price goes up even further the investor starts buying again as he becomes risk neutral until he attains satiation and is willing to sell again.

How do skewness and kurtosis affect the optimal investment strategy? In the case of constant relative risk aversion or for mean variance optimizers, skewed and fat-tailed return distributions do not affect the optimal allocation to stocks. When employing downside-risk measures this is no longer true. We find that the impact of skewness and kurtosis is quite large and should not be ignored. Again we find striking results: expected loss and probability of loss minimizers $(0 \leq \gamma \leq 1)$ increase the stock weight as the return distribution becomes more negatively skewed. 
This can be understood by realizing that investors caring mainly about falling below the target $(0 \leq \gamma \leq 1)$ become risk seeking when confronted with gains. When the investor cares more about the magnitude of wealth below the target $(\gamma>1)$ skewness and kurtosis mainly effect the optimal strategy at large levels of wealth (due to risk neutrality).

In conclusion, despite the popularity of downside-risk as a measure for investment risk and a guideline for optimal investment strategies, a mean versus downside-risk framework implies many peculiarities:

1. Both at low and high levels of wealth the investor gambles by investing more in risky assets;

2. Downside-risk aversion implies reversed time effects: confronted with a shorter horizon the investor invests more in risky assets;

3. Confronted with negatively skewed and fat-tailed returns the investor invests more in risky assets.

These results can be understood by realizing that:

1. When confronted with gains the investor becomes risk neutral, and hence increases his exposure to stocks;

2. When confronted with losses the investor is either risk seeking $(0 \leq \gamma<1)$ or displays increasing relative risk aversion $(\gamma=2)$, and hence desires to gamble by investing more in stocks.

Downside-risk measures may be useful as a summary statistic for decision-makers, however as a tool for managing and controlling risk it has severe drawbacks as we have demonstrated in this paper. Investors and practitioners should be aware of the gambling effects prevalent in the optimal policies under downside risk aversion. Although short selling constraints and position limits will prevent investors in practice to accept extremely risky investments, the optimal asset allocation will certainly be driven by the effects found in this paper.

A possible remedy to the consequences of the mean-downside risk framework studied in this paper, might be to consider optimal portfolio choice with power utility over wealth and additional side constraints restricting the downside risk of the portfolio. This avoids the drawbacks of risk neutrality above the target. However, the analysis of Basak \& Shapiro (1999) in an expected utility framework with value-at-risk as a side constraint, demonstrates that the optimal policies still exhibit gambling effects.

\section{References}

Basak, S. \& Shapiro, A. (1999), Value-at-risk based risk management: Optimal policies and asset prices, Working paper, The Wharton School, University of Pennsylvania.

Bawa, V. \& Lindenberg, E. (1977), 'Capital market equilibrium in a mean-lower partial moment framework', Journal of Financial Economics 5, 189-200. 
Brennan, M., Schwartz, E. \& Lagnado, R. (1997), 'Strategic asset allocation', Journal of Economic Dynamics and Control 21, 1377-1403.

Campbell, J. \& Viceira, L. (1999), 'Consumption and portfolio decisions when expected returns are time-varying', Quarterly Journal of Economics 114, 433-495.

Cox, J. \& Huang, C. (1989), 'Optimum consumption and portfolio policies when asset prices follow a diffusion process', Journal of Economic Theory 49, 33-83.

Dert, C. \& Oldenkamp, K. (1997), Optimal guaranteed return portfolios and the casino effect, Working paper, Econometric Institute, Erasmus University Rotterdam.

Dybvig, P. (1995), 'Duesenberry's ratcheting of consumption: Optimal dynamic consumption and investment given intolerance for any decline in standard of living', Review of Economic Studies 62, 287-313.

Eberlein, E., Keller, U. \& Prause, K. (1998), 'New insights into smile, mispricing and value at risk: the hyperbolic model', Journal of Business 71, 371-405.

Fama, E. (1965), 'The behavior of stock prices', Journal of Business 47, 244-280.

Fishburn, P. (1977), 'Mean-risk analysis with risk associated with below-target returns', American Economic Review pp. 116-125.

Harlow, W. (1991), 'Asset allocation in a downside risk framework', Financial Analyst Journal 47(5), 28-40.

Jarrow, R. \& Rudd, A. (1982), 'Approximate option valuation for arbitrary stochastic processes', Journal of Financial Economics 10, 347-369.

Jorion, P. (1997), Value at Risk: The New Benchmark for Controlling Market Risk, Irwin Professional.

Kahneman, D. \& Tversky, A. (1979), 'Prospect theory: An analysis of decision under risk', Econometrica 47, 263-290.

Karatzas, I. \& Shreve, S. (1998), Methods of Mathematical Finance, Springer Verlag.

Kim, T. \& Omberg, E. (1996), 'Dynamic nonmyopic portfolio behavior', Review of Financial Studies 9, 141-161.

Kritzman, M. (1998), 'Beware of dogma', Journal of Portfolio Management 24(4), 66-78.

Longstaff, F. (1995), 'Option pricing and the martingale restriction', Review of Financial Studies 8, 1091-1124.

Madan, D. \& Seneta, E. (1990), 'The variance gamma model for share market returns', Journal of Business 63(4), 511-524.

Markowitz, H. (1952), 'Portfolio selection', Journal of Finance 7, 77-91.

Markowitz, H. (1959), Portfolio Selection, Efficient Diversification of Investments, Cowles Foundation for Research in Economics at Yale University, Monograph 16, John Wiley \& Sons, New York, USA.

Markowitz, H., Todd, P., Xu, G. \& Yamane, Y. (1993), 'Computation of mean-semivariance efficient sets by the critical line algorithm', Annals of Operations Research 45, 307-317.

Merton, R. (1969), 'Lifetime portfolio selection under uncertainty: The continuous-time case', Review of Economics and Statistics 51, 247-257. 
Merton, R. (1990), Continuous Time Finance, Basil Blackwell, Oxford.

Samuelson, P. (1969), 'Lifetime portfolio selection by dynamic stochastic programming', Review of Economics and Statistics 51, 239-246.

Sørensen, C. (1999), 'Dynamic asset allocation and fixed income management', Journal of Financial and Quantitative Analysis 34(4).

Sortino, F. \& van der Meer, R. (1991), 'Downside risk', Journal of Portfolio Management Summer, 27-31. 


\section{A Mathematical Proofs}

\section{Proof of Proposition 2}

Problem (8) is a convex optimization problem with a concave objective function. We rely on the convex-duality approach (see, e.g. Karatzas \& Shreve (1998)). The Legendre-Fenchel transform for (8) is defined by

$$
U^{*}\left(\xi_{T}\right)=\max _{W \geq 0}\left\{U(W)-y \xi_{T} W\right\}
$$

where $\xi_{T} \geq 0$ denotes the pricing kernel. We first consider the solution to this pointwise maximization problem for all $\xi_{T}$, and show that (10) solves this problem. Then, we show that the solution to the pointwise maximization problem (20) also solves (8).

Let us denote the part of the utility function below $\theta$ by $U_{1}(W)$ and the part above $\theta$ by $U_{2}(W)$. If $W \leq \theta$, the optimally invested wealth $W^{*}$ should satisfy the following Karush-Kuhn-Tucker (KKT) conditions

$$
\begin{aligned}
U_{1}^{\prime}\left(W^{*}\right) & =y \xi_{T}-\lambda, W^{*} \geq 0 \\
\lambda W^{*} & =0, \lambda \geq 0
\end{aligned}
$$

where $\lambda$ denotes the Lagrange multiplier associated with the nonnegativity constraint on wealth. Solving for the KKT conditions we obtain

$$
W^{*}=\max \left\{\theta-\left(\frac{A \gamma}{y \xi_{T}}\right)^{1 / 1-\gamma}, 0\right\}:=W_{1}^{*},
$$

when $\xi_{T} \leq 1 / y$. If $W \geq \theta$, the function $U(W)-y \xi_{T} W$ is linear. Consequently, the optimally invested wealth satisfies

$$
W_{2}^{*}= \begin{cases}\bar{W} & \text { if } \xi_{T}<a / y \\ \theta & \text { if } \xi_{T}>a / y\end{cases}
$$

When $\xi_{T}<\frac{a}{y}$ the optimal solution is obviously given by $W_{2}^{*}=\bar{W}$. In case $\xi_{T} \geq 1 / y$, the solution is given by $W_{1}^{*}$. Let us compare the objective values of $W_{1}^{*}$ and $W_{2}^{*}$. If

$$
U\left(W_{1}^{*}\right)-y \xi_{T} W_{1}^{*} \geq U\left(W_{2}^{*}\right)-y \xi_{T} W_{2}^{*}
$$

the optimal solution is given by $W_{1}^{*}$. It is not difficult to verify that $W_{1}^{*}$ is indeed the optimal solution for $\gamma>1$ when $\xi_{T} \geq \frac{1}{y}$.

Let us denote the optimal solution of $(20)$ by $W^{*}(T)$. Now let $W(T)$ be any candidate optimal solution, satisfying the static budget equation in (8). Then, we have

$$
\begin{array}{ll}
E\left[U\left(T, W_{T}^{*}\right)\right]-E\left[U\left(T, W_{T}\right)\right] & = \\
E\left[U\left(T, W_{T}^{*}\right)\right]-E\left[U\left(T, W_{T}\right)\right]-y \xi_{0} W_{0}+y \xi_{0} W_{0} & \geq \\
E\left[U\left(T, W_{T}^{*}\right)\right]-E\left[U\left(T, W_{T}\right)\right]-y E\left[\xi_{T} W_{T}^{*}\right]+y E\left[\xi_{T} W_{T}\right] & = \\
E\left[U^{*}\left(\xi_{T}\right)\right]-E\left[U^{*}\left(\xi_{T}\right)\right] & \geq 0
\end{array}
$$


where the first inequality follows from the fact that the static budget equation holds with equality for $W_{T}^{*}$ and with inequality for $W_{T}$. The second inequality follows from the fact that $W_{T}^{*}$ is the optimal solution to (20). Hence, we conclude that $W^{*}(T)$ is the optimal solution of the static problem (8) with utility (6) when $\gamma>1$. This concludes the proof.

\section{Proof of Proposition 1}

The proof of this proposition follows similar arguments as the proof of Proposition 2. Consider the pointwise problem

$$
U^{*}\left(\xi_{T}\right)=\max _{W \geq 0}\left\{U(W)-y \xi_{T} W\right\}
$$

where $\xi_{T} \geq 0$ denotes the pricing kernel.

Let us denote the part of the utility function below $\theta$ by $U_{1}(W)$ and the part above $\theta$ by $U_{2}(W)$. If $W \geq \theta$, the function $U(W)-y \xi_{T} W$ is linear. Consequently, the optimally invested wealth satisfies

$$
W_{2}^{*}= \begin{cases}\bar{W} & \text { if } \xi_{T}<\frac{1}{y} \\ \theta & \text { if } \xi_{T}>\frac{1}{y}\end{cases}
$$

If $W \leq \theta$, the utility function is convex. In a maximization problem the corresponding optimal solution with a convex objective is always a corner solution, i.e. either $W_{1}^{*}=\theta$ or $W_{1}^{*}=0$. To determine the optimum when $W \leq \theta$ we compare the objective values of $W_{1}^{*}=\theta$ and $W_{1}^{*}=0$. It is not difficult to verify that

$$
W_{1}^{*}= \begin{cases}\theta & \text { if } \frac{1}{y} \leq \xi_{T} \leq \frac{1}{y}+\frac{A}{y} \theta^{\gamma-1} \\ 0 & \text { if } \xi_{T}>\frac{1}{y}+\frac{A}{y} \theta^{\gamma-1}\end{cases}
$$

A similar argument as in the proof of Proposition 2 shows that (9) is the optimal solution to (8) for utility function (6) with $0 \leq \gamma<1$. A separate analysis shows that (9) is also the optimal solution to (8) when $\gamma=1$. This concludes the proof.

\section{Proof of Proposition 3}

(i) Applying Ito's Lemma it is straightforward to show that $\xi_{t} W_{t}$ is a martingale. Therefore

$$
W_{t}=\frac{1}{\xi_{t}} E_{t}\left[\xi_{T} W_{T}\right]=\frac{1}{\xi_{t}} E_{t}\left[\xi_{T}\left(\bar{W} \mathbf{1}_{\left\{\xi_{T} \leq \underline{\xi}\right\}}+\theta \mathbf{1}_{\left\{\underline{\xi} \leq \xi_{T} \leq \bar{\xi}\right\}}\right)\right],
$$

where we substituted (9). Since $r$ and $\kappa$ are constant, and the asset prices follow geometric Brownian motions, the pricing kernel $\log \left(\xi_{T}\right)$ is normally distributed with mean

$$
\log \left(\xi_{t}\right)-\left(r+\frac{1}{2}\|\kappa\|^{2}\right)(T-t)
$$

and variance $\|\kappa\|^{2}(T-t)$. After some straightforward calculus we obtain (11a). 
(ii) We have two alternative characterizations for wealth at time $t$. Reformulating (11a) as a stochastic process and equating the diffusion part of this stochastic process with the diffusion part of the wealth process (2) we obtain an explicit expression for the fraction of wealth invested in the risky assets.

For ease of notation we define wealth as $W_{t}=: F\left(t, \xi_{t}\right)$. Using Ito's Lemma and (3) we obtain:

$$
d W_{t}=G\left(t, \xi_{t}\right) d t-\frac{\partial F\left(t, \xi_{t}\right)}{\partial \xi_{t}} \xi_{t} \kappa^{\prime} d B_{t}
$$

for some $G\left(t, \xi_{t}\right)$ (note that we are only interested in the diffusion part). Comparing the diffusion part of (2) with the diffusion part of (24) we obtain the following expression for the optimal fraction invested in the risky assets:

$$
w_{t}=-\frac{\left(\sigma^{\prime}\right)^{-1} \kappa^{\prime}}{W_{t}}\left(\frac{\partial F\left(t, \xi_{t}\right)}{\partial \xi_{t}} \xi_{t}\right) .
$$

Substituting (11a) in (25) yields the expression in (12).

\section{Proof of Proposition 4}

The proof is completely analogous to the proof of Proposition 3, using (10).

\section{Proof of Proposition 5}

(i) The proof is analogous to the proof of part (i) in Proposition 3, except that the pricing kernel is not lognormally distributed. As we used a Gram-Charlier expansion of the lognormal density function, the pricing kernel $\log \left(\xi_{T}\right)$ has the following density function:

$$
f\left(z_{T} ; \beta, k\right)=\left(1+\beta\left(z_{T}^{3}-3 z_{T}\right)+\delta\left(z_{T}^{4}-6 z_{T}^{2}+3\right)\right) \phi\left(z_{T}\right),
$$

where $z_{T}=\left(\log \left(\xi_{T}\right)-\mu\right) / \sigma, \beta=\zeta / 6, \delta=\chi / 24$ and $\zeta, \chi$ denote skewness and kurtosis of $\log \left(\xi_{T}\right)$ respectively, and $\mu=\log \left(\xi_{t}\right)-\left(r+\frac{1}{2}\|\kappa\|^{2}\right)(T-t), \sigma=\|\kappa\| \sqrt{T-t}$ denote the mean and standard deviation of $\log \left(\xi_{T}\right)$. After some tedious but straightforward calculus we obtain (16).

(ii) The proof is analogous to the proof of part (ii) in Proposition 3. Denote wealth by $W_{t}=$ $F\left(t, \xi_{t}\right)$. The optimal fraction invested in the risky assets is given by:

$$
w_{t}=-\frac{\left(\sigma^{\prime}\right)^{-1} \kappa^{\prime}}{W_{t}}\left(\frac{\partial F\left(t, \xi_{t}\right)}{\partial \xi_{t}} \xi_{t}\right)
$$

Substituting (16) in (26) yields the expression in (17).

\section{Proof of Proposition 6}

The proof is completely analogous to the proof of Proposition 5, using (10). 

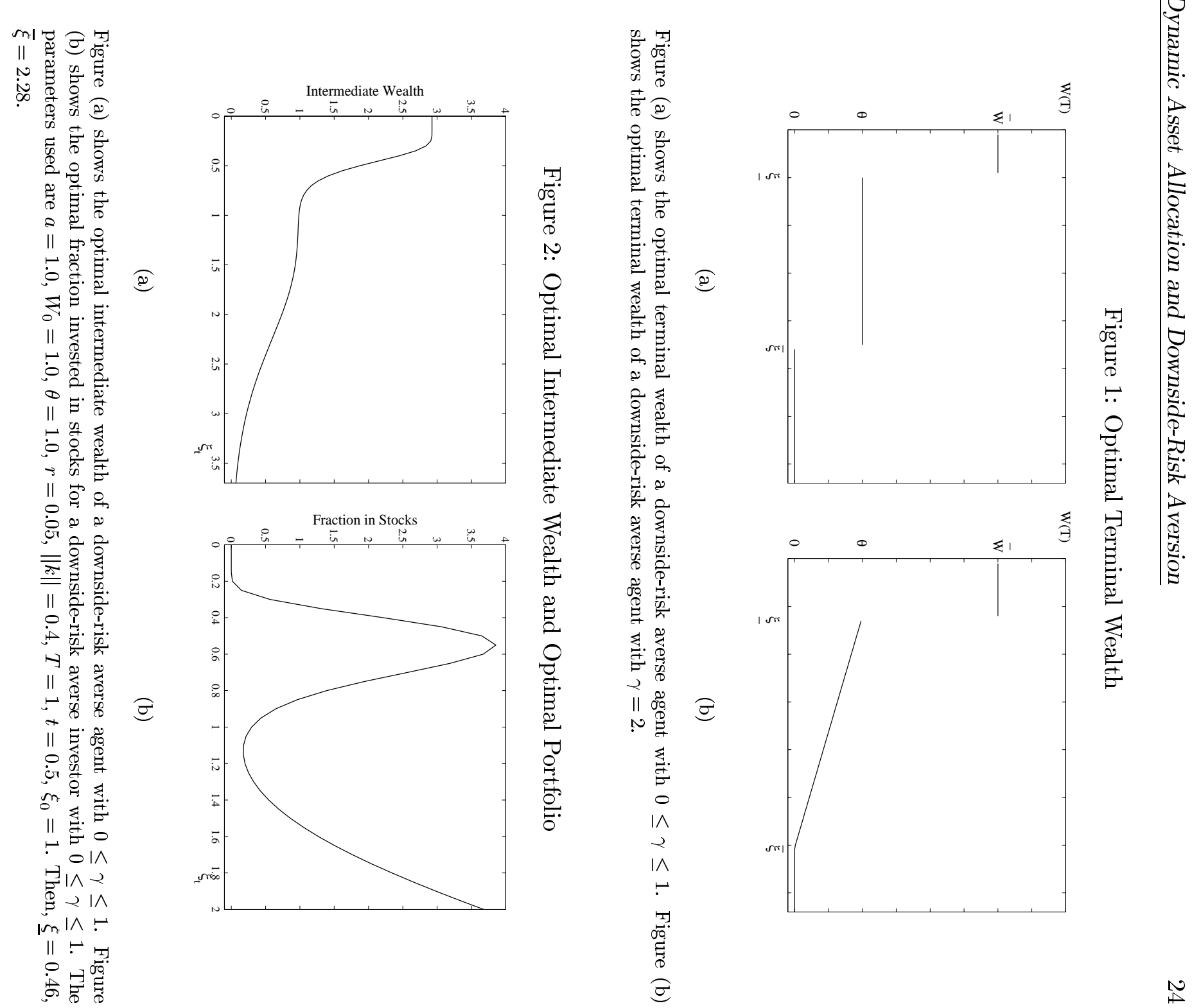
Figure 3: Optimal Intermediate Wealth and Optimal Portfolio

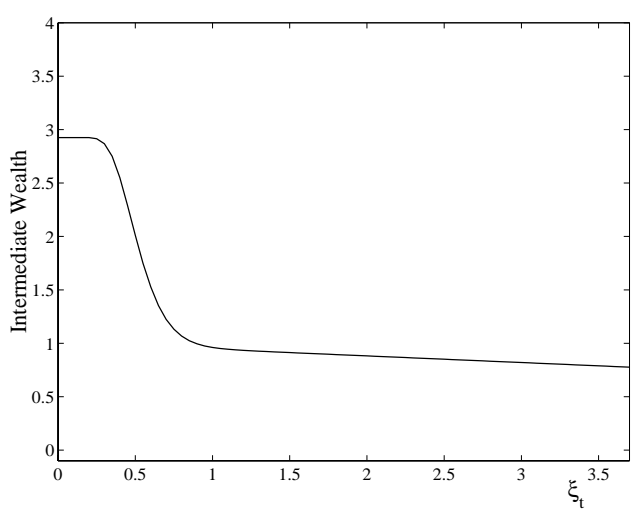

(a)

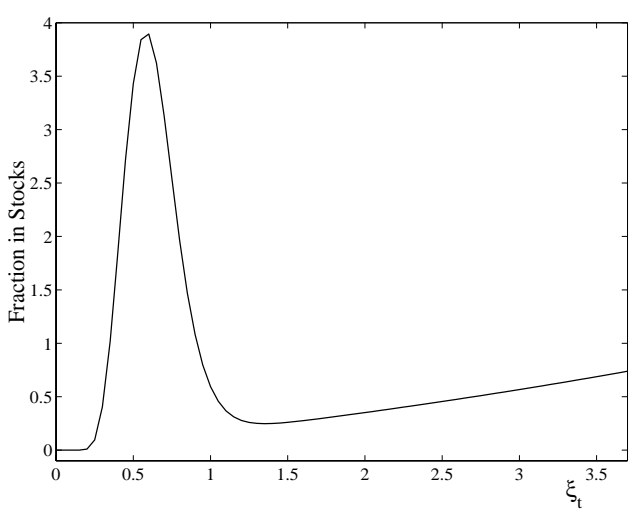

(b)

Figure (a) shows the optimal intermediate wealth of a downside-risk averse agent with $\gamma=2$. Figure (b) shows the optimal fraction invested in stocks for a downside risk averse investor with $\gamma=2.0$. The parameters used are $A=16.0, W_{0}=1.0, \theta=1.0, r=0.05,\|k\|=0.4, T=1, t=0.5, \xi_{0}=1$. Then, $\underline{\xi}=0.52, \bar{\xi}=17.15$.

Figure 4: Optimal Fraction Invested (Feedback Form)

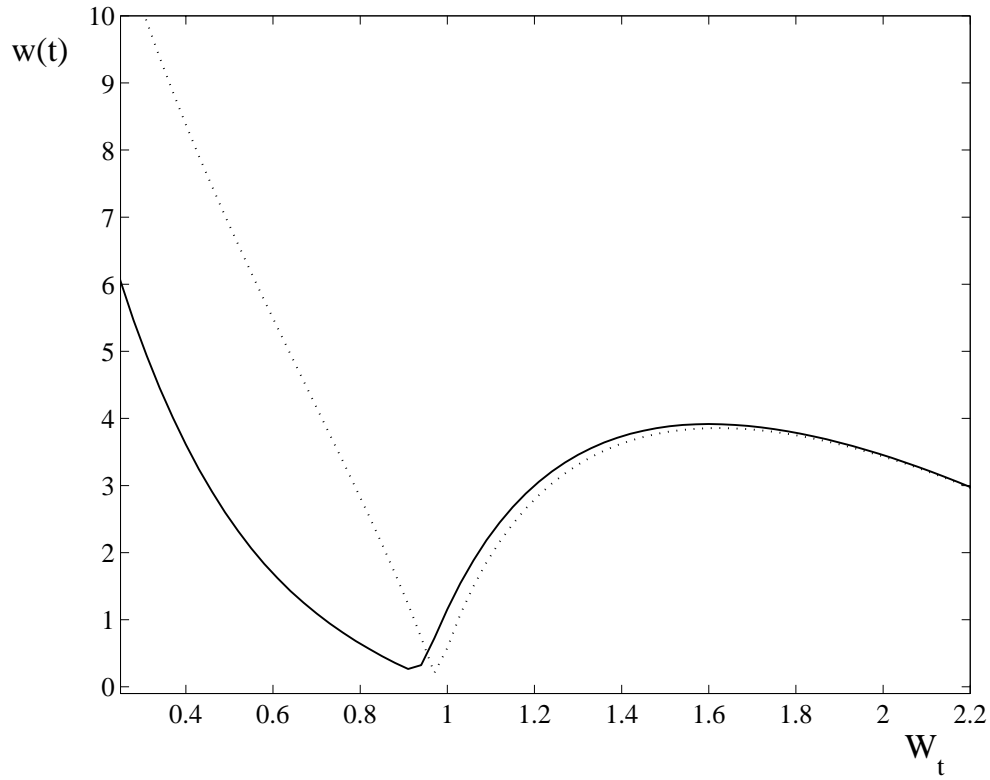

This figure shows the optimal fraction invested as a function of wealth for a downside-risk averse agent with $0 \leq \gamma \leq 1$ (dotted plot) and for a downside-risk averse investor with $\gamma=2$ (solid plot). The parameters used are $W_{0}=1.0, \theta=1.0, r=0.05,\|k\|=0.4, T=1, t=0.5, \xi_{0}=1$. 
Figure 5: Indirect Relative Risk Aversion Function

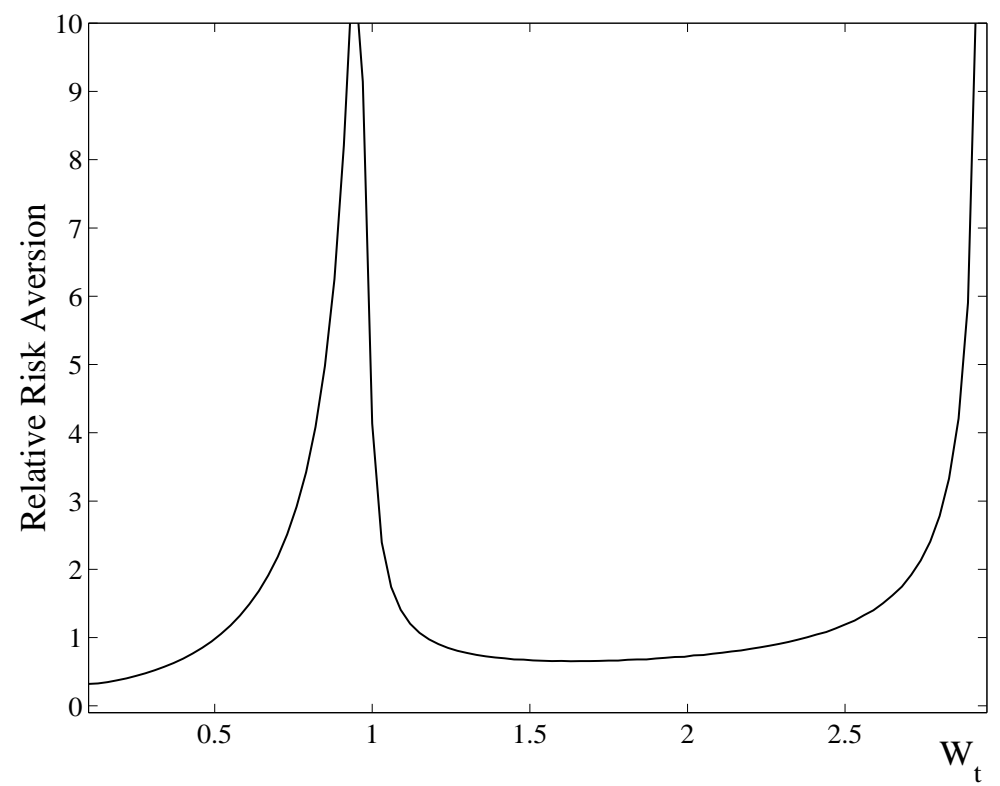

This figure shows relative risk aversion function of the indirect utility function for a downside-risk averse agent with $\gamma=2$. The parameters used are $A=16.0, W_{0}=1.0, \theta=1.0, r=0.05,\|k\|=0.4, T=1, t=0.5$, $\xi_{0}=1$.

Figure 6: Optimal Fraction Invested: Time Effects

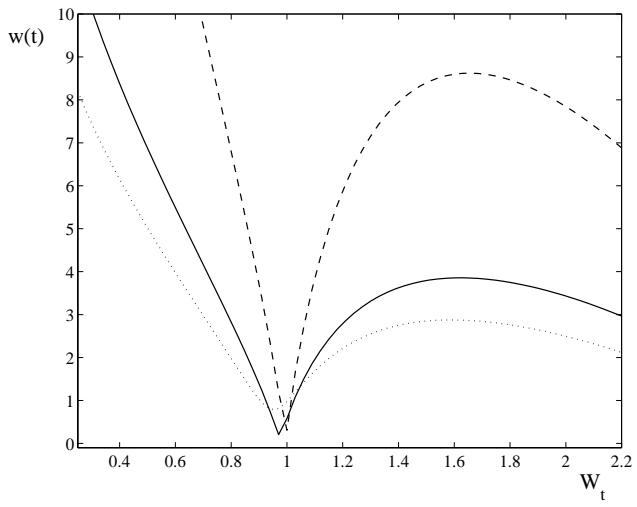

(a)

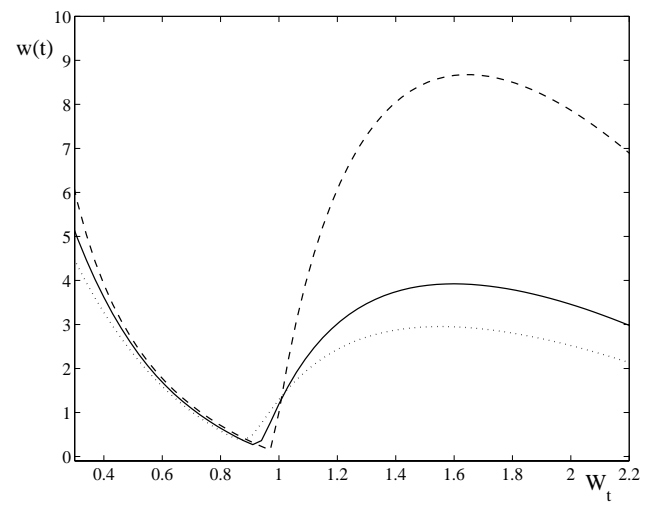

(b)

Figure (a) shows the time effects for a downside-risk averse agent with $0 \leq \gamma \leq 1, t=0.1$ (dotted plot), $t=0.5$ (solid plot), and $t=0.9$ (dashed plot). Figure (b) shows the time effects for a downside-risk averse investor with $\gamma=2, t=0.1$ (dotted plot), $t=0.5$ (solid plot), and $t=0.9$ (dashed plot). The parameters used are $W_{0}=1.0, \theta=1.0, r=0.05,\|k\|=0.4, T=1, \xi_{0}=1$. 
Figure 7: Number of Stocks Purchased

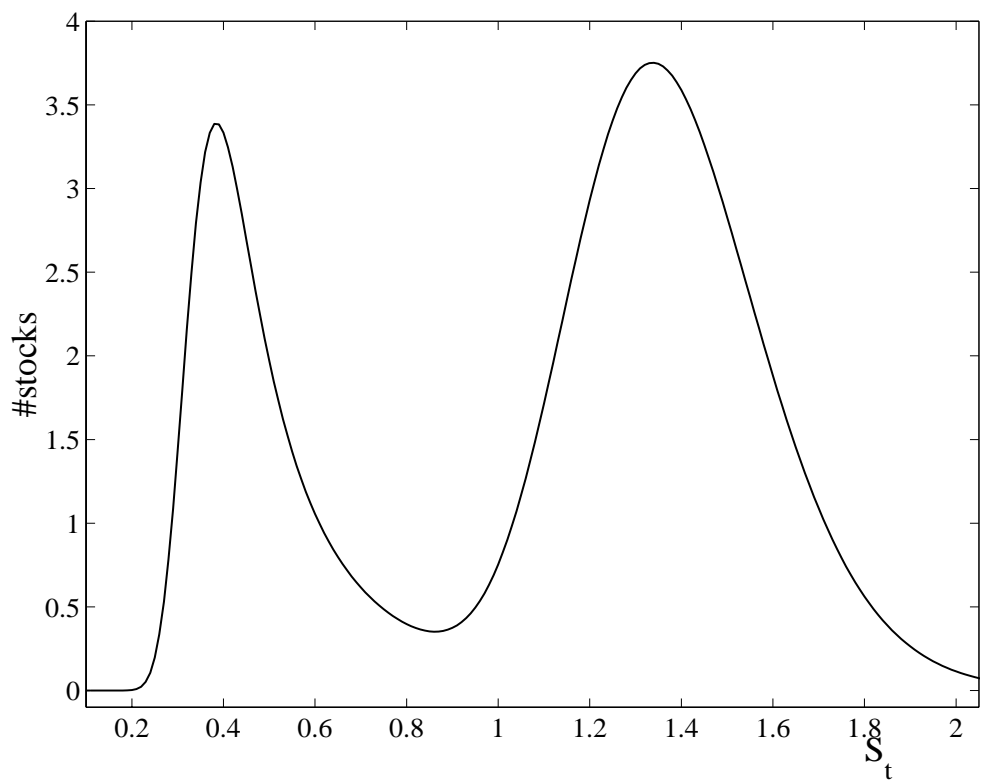

This figure shows the number of stocks purchased as a function of the stock price for a downside-risk averse agent with $\gamma=2$. The parameters used are $W_{0}=1.0, \theta=1.0, r=0.05,\|k\|=0.4, T=1, t=0.5, \xi_{0}=1$. Then, $\xi_{1}=, \xi_{2}=$.

Figure 8: Optimal Fraction Invested: Impact of Skewness and Kurtosis

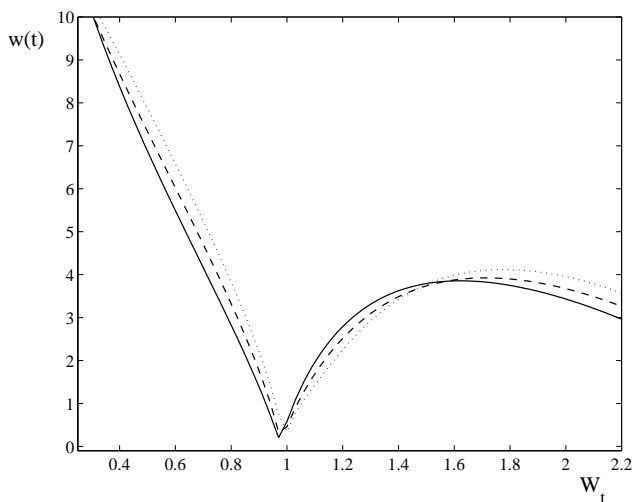

(a)

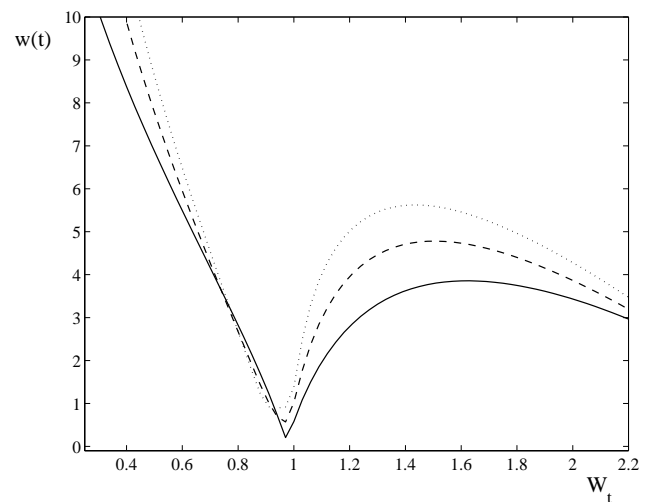

(b)

Figure (a) shows the effects of negative skewness for a downside-risk averse agent with $0 \leq \gamma \leq 1, \zeta=0.0$ (solid plot), $\zeta=-0.4$ (dashed plot), and $\zeta=-0.8$ (dotted plot). Figure (b) shows the effects of excess kurtosis for a downside-risk averse investor with $0 \leq \gamma \leq 1, \chi=0.0$ (solid plot), $\chi=1.2$ (dashed plot), and $\chi=2.2$ (dotted plot). The parameters used are $W_{0}=1.0, \theta=1.0, r=0.05,\|k\|=0.4, T=1, \xi_{0}=1$. 
Figure 9: Optimal Fraction Invested: Impact of Skewness and Kurtosis

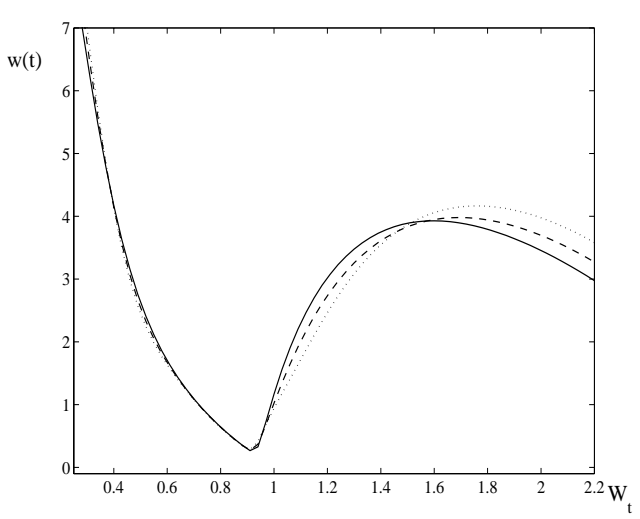

(a)

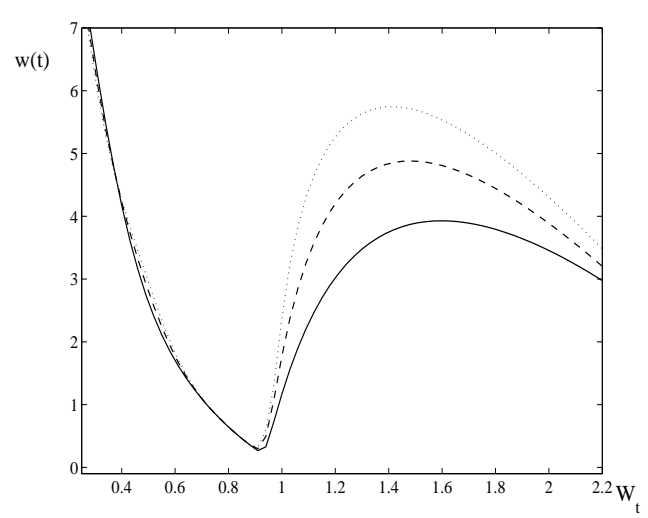

(b)

Figure (a) shows the effects of negative skewness for a downside-risk averse agent with $\gamma=2, \zeta=0.0$ (solid plot), $\zeta=-0.4$ (dashed plot), and $\zeta=-0.8$ (dotted plot). Figure (b) shows the effects of excess kurtosis for a downside-risk averse investor with $\gamma=2, \chi=0.0$ (solid plot), $\chi=1.2$ (dashed plot), and $\chi=2.2$ (dotted plot). The parameters used are $W_{0}=1.0, \theta=1.0, r=0.05,\|k\|=0.4, T=1, \xi_{0}=1$. 